\title{
Constraining the era of helium reionization using fast radio bursts
}

DOI:

$10.1093 /$ mnras/stz571

\section{Document Version}

Accepted author manuscript

Link to publication record in Manchester Research Explorer

\section{Citation for published version (APA):}

Caleb, M., Flynn, C., \& Stappers, B. (2019). Constraining the era of helium reionization using fast radio bursts. Monthly Notices of the Royal Astronomical Society. https://doi.org/10.1093/mnras/stz571

\section{Published in:}

Monthly Notices of the Royal Astronomical Society

\section{Citing this paper}

Please note that where the full-text provided on Manchester Research Explorer is the Author Accepted Manuscript or Proof version this may differ from the final Published version. If citing, it is advised that you check and use the publisher's definitive version.

\section{General rights}

Copyright and moral rights for the publications made accessible in the Research Explorer are retained by the authors and/or other copyright owners and it is a condition of accessing publications that users recognise and abide by the legal requirements associated with these rights.

\section{Takedown policy}

If you believe that this document breaches copyright please refer to the University of Manchester's Takedown Procedures [http://man.ac.uk/04Y6Bo] or contact uml.scholarlycommunications@manchester.ac.uk providing relevant details, so we can investigate your claim.

\section{OPEN ACCESS}




\title{
Constraining the era of helium reionization using fast radio bursts
}

\author{
M. Caleb ${ }^{1 \star}$, C. Flynn ${ }^{2,3}$, B.W. Stappers ${ }^{1}$ \\ ${ }^{1}$ Jodrell Bank Centre for Astrophysics, School of Physics and Astronomy, The University of Manchester, Manchester M13 9PL, UK \\ ${ }^{2}$ Centre for Astrophysics and Supercomputing, Swinburne University of Technology, P.O. Box 218, Hawthorn, VIC 3122, Australia \\ ${ }^{3}$ ARC Centre of Excellence for All-sky Astrophysics (CAASTRO)
}

Accepted XXX. Received YYY; in original form ZZZ

\begin{abstract}
The discovery of fast radio bursts (FRBs) about a decade ago opened up new possibilities for probing the ionization history of the Intergalactic Medium (IGM). In this paper we study the use of FRBs for tracing the epoch of HeII reionization, using simulations of their dispersion measures. We model dispersion measure contributions from the Milky Way, the IGM (homogeneous and inhomogeneous) and a possible host galaxy as a function of redshift and star formation rate. We estimate the number of FRBs required to distinguish between a model of the Universe in which helium reionization occuured at $z=3$ from a model in which it occurred at $z=6$ using a 2-sample Kolmogorov-Smirnoff test. We find that if the IGM is homogeneous $\gtrsim 1100$ FRBs are needed and that an inhomogeneous model in which traversal of the FRB pulse through galaxy halos increases the number of FRBs modestly, to $\gtrsim 1600$. We also find that to distinguish between a reionization that occurred at $z=3$ or $z=3.5$ requires $\gtrsim 5700$ FRBs in the range $3 \leq z \leq 5$.
\end{abstract}

Key words: radio continuum: transients - cosmology: miscellaneous - keyword3

\section{INTRODUCTION}

One of the key questions in present day cosmology is when the reionization of Helium occurred. Following cosmological recombination at redshift $z \sim 10^{3}$, the baryonic gas in the Universe remained primarily neutral. Given that presently most of the observable baryonic gas is ionized, phase transitions of this gas must have taken place at some redshift $z<10^{3}$. The progress of reionization is regulated by the ioinizing radiation escaping the host galaxies from e.g. quasars and stars. Reionization typically refers to the events related to neutral hydrogen (HI) and helium (HeI) at $z \sim 6$ when the atoms lost their first outer electrons and marked an important change in the structure of the Universe known as the Epoch of Reionization (EoR; Fan et al. 2002; Zaroubi 2013; Singh et al. 2018). Present observational constraints on the EoR have mostly been indirect but direct observations of the redshifted $21-\mathrm{cm}$ signal from neutral hydrogen using present and upcoming experiments such as the Low-Frequency Array (LOFAR; van Haarlem et al. 2013), Murchison Wide-field array (MWA; Tingay et al. 2013), Precision Array for Probing the Epoch of Reionization (PAPER; Parsons et al. 2014) and the Hydrogen Epoch of Reionization Array (HERA; DeBoer et al. 2017) are expected to provide stronger constraints.

Following the EoR, the high ionization potential $(54.4 \mathrm{eV})$ of singly ionised helium (HeII) prevented it from being further reionized until a sufficient build-up of hard-spectra quasars provided enough photons to complete the reionization. HeII is expected to have undergone a second reionization at $z \sim 3$ with significant

^ Email: manisha.caleb@manchester.ac.uk contributions from stars and quasars leaving an impression on the IGM. The strongest evidence of this is seen through observational signatures in the far ultraviolet spectra of the HeII Ly $\alpha$ forest along the lines-of-sight to several quasars at $z \sim 3$ (e.g. Syphers et al. 2009, 2012). The Ly $\alpha$ transition for neutral hydrogen, Hi exhibits as absorption features in the spectra of distant quasars just as it does for the transition of singly ionized helium, HeIr. However observations of the HeII Ly $\alpha$ forest for the second reionization is mostly obscured by contamination due to the relative abundance of high-density systems at low redshifts (see La Plante et al. 2017, and references therein). The comparatively low number of lines-of-sight that show the Ly $\alpha$ forest signature for HeIr leaves much statistical uncertainly about the exact timing and nature of the reionization process. Other evidence of reionization is the temperature evolution of the intergalactic medium (IGM) with a quick change at $z \sim 3$ (McQuinn et al. 2009). The comparatively lower redshift of HeII reionization makes it more accessible to observations.

FRBs are intense bursts ( Jy) of coherent emission of unknown origin, lasting only a few milliseconds. Over the last decade or so, realization has grown that this and other properties could be used as a cosmological probe (see Keane (2018) for a recent review). In particular, their short durations enable direct measurements of the integrated column densities of ionized plasma known as dispersion measures (DMs), along the observed lines-of-sight thereby tracing all the ionized baryons along its propagation path. Electron density models of the Milky Way (e.g. Cordes \& Lazio 2003; Yao et al. 2017) are unable to account for more than a few percent of the total observed DM values, thereby suggesting extragalactic/cosmological origins for FRBs. Precise localization to a 
host galaxy is however necessary to determine the true extragalactic or cosmological nature of FRBs, as was demonstrated for FRB 121102 (Chatterjee et al. 2017; Tendulkar et al. 2017). FRBs with redshifts obtained from host galaxies could be used to probe the epoch of helium reionization (Deng \& Zhang 2014; Zheng et al. 2014), map the intergalactic magnetic fields (Macquart et al. 2015), locate the 'missing' baryons in the Univers (McQuinn 2014) and study the dark energy equation-of-state (Zhou et al. 2014).

In this letter we study the possibility of probing the epoch of HeII reionization with FRBs through simulations of their observed DMs and estimate the numbers of FRBs required, assuming they have been localised to a host galaxy with a measured redshift. We present our DM model for the simulated FRBs in Section 2 followed by our analyses and results in Section 3 . We finally present our discussion and conclusions in Section 4.

\section{MODELING DISPERSION MEASURES}

The DM of an FRB parameterises the delay in arrival time as a function of frequency of a pulse which is given by,

$\Delta t=4.15 \mathrm{~ms} \times\left[\left(\frac{v_{1}}{\mathrm{GHz}}\right)^{-2}-\left(\frac{v_{2}}{\mathrm{GHz}}\right)^{-2}\right] \times\left(\frac{\mathrm{DM}}{\mathrm{pc} \mathrm{cm}^{-3}}\right)$

and is an observable quantity. This delay in arrival time, in the observer's frame is $\Delta t=\Delta t^{\prime}(1+z)$, where $\Delta t^{\prime}$ is the rest-frame delay. Similarly, the observed frequencies in the observer's frame are $v_{1}=v_{1}^{\prime}(1+z)$ and $v_{2}=v_{2}^{\prime}(1+z)$ with $v_{1}^{\prime}$ and $v_{2}^{\prime}$ being the source rest-frame frequencies. Using these relations, the DM measured in the observer's frame in Equation 1 is related to the free electron density along the line-of-sight as,

$\mathrm{DM}=\int_{0}^{z} \frac{n_{\mathrm{e}}}{(1+z)} d l$

For an FRB originating at cosmological distances, the observed DM value can be expressed as the sum of several contributions along the path traversed given by,

$\mathrm{DM}_{\text {obs }}=\mathrm{DM}_{\mathrm{MW}}+\mathrm{DM}_{\mathrm{IGM}}+\frac{\mathrm{DM}_{\text {host }}+\mathrm{DM}_{\text {source }}}{(1+z)}$

where $\mathrm{DM}_{\mathrm{MW}}$ is the contribution from the Milky Way (MW), $\mathrm{DM}_{\mathrm{IGM}}$ is the contribution from the diffuse homogeneous/inhomogeneous IGM along the line-of-sight, $\mathrm{DM}_{\text {host }}$ is any contribution from the interstellar medium (ISM) of the host galaxy and $\mathrm{DM}_{\text {source }}$ is the contribution from the immediate vicinity of the FRB progenitor. The lack of understanding of the physical origins of FRBs, hinders our ability to ascertain the $\mathrm{DM}_{\text {host }}$ and $\mathrm{DM}_{\text {source }}$ components of the total observed DM reliably. However due to the cosmological redshifting of frequency, their combined contribution is diluted by a factor of $(1+z)$ to the Earth observer from the restframe observer (Ioka 2003; Deng \& Zhang 2014) and is likely to be small. As a result the $\mathrm{DM}_{\mathrm{IGM}}$ is the dominant contribution to the observed DM as the number density of free electrons scales as $(1+z)^{3}$.

\subsection{DM contribution from the Milky Way}

The MW component of the total observed DM can be reasonably estimated using either the NE2001 (Cordes \& Lazio 2003) or the
YMW16 model (Yao et al. 2017) and removed from the data with modest uncertainties. Both models use Galactic pulsars to map the the integrated electron density along any given line-of-sight through the MW which strongly decreases as a function of Galactic latitude $|b|$ from $\sim 10^{3} \mathrm{pc} \mathrm{cm}^{-3}$ near the Galactic centre to an average of $\sim 100 \mathrm{pc} \mathrm{cm}^{-3}$ at $10^{\circ} \leq|b| \leq 40^{\circ}$. It should be noted that the estimate of DM at $|b|>40^{\circ}$ are less reliable due to the lack of pulsars in the Galactic halo. Since the electron density content in the Galactic halo is relatively low with a correspondingly small DM contribution $\left(\sim 30 \mathrm{pc} \mathrm{cm}^{-3}\right)$ as seen from simulations by Dolag et al. (2015), we do not include a term for the halo contribution in Equation 3. Overall, for an extragalactic source like an FRB, the contribution from the Milky Way is relatively small over most of the sky, and can be avoided in FRB searches if needed.

\subsection{DM contribution from IGM}

The free electron density at any given redshift can be expressed as (Deng \& Zhang 2014),

$n_{e}=\frac{\rho_{c, 0} \Omega_{\mathrm{b}} f_{\mathrm{IGM}} f_{e}(z)}{m_{p}} \times(1+z)^{3}$,

where $\rho_{c, 0}$ is the critical mass density, $\Omega_{b}=0.049$ is the present baryon density of the Universe (Planck Collaboration et al. 2016) and,

$f_{e}(z)=\frac{3}{4} \chi_{e, \mathrm{H}}(z)+\frac{1}{8}\left[\chi_{e, \mathrm{He}}(z)+\chi_{e, \mathrm{He} I I I}(z)\right]$,

with the terms $\chi_{e, \mathrm{H}}(z)$ and $\chi_{e, \mathrm{He}}(z)$ representing the ionization mass fractions of hydrogen and helium respectively. Strictly speaking, the baryon mass fraction in the IGM, $f_{\text {IGM }}$ varies slightly a function of redshift from 0.9 at $z \gtrsim 1.5$ to 0.82 at $z \leq 0.4$ (Shull et al. 2012). To first order, we assume it to be a constant at 0.83 (Fukugita et al. 1998; Shull et al. 2012). For a path length $d l$ given by (Deng \& Zhang 2014),

$d l=\frac{1}{1+z} \frac{c}{H_{0}} \frac{d z}{\sqrt{\Omega_{m}(1+z)^{3}+\Omega_{\Lambda}}}$,

where the matter density $\Omega_{m}=0.308$, the vacuum density $\Omega_{\Lambda}=$ 0.6911 and Hubble constant $H_{0}=67.74 \mathrm{~km} \mathrm{~s}^{-1} \mathrm{Mpc}^{-1}$ (Planck Collaboration et al. 2016), we can combine Equations 4 and 6 to obtain a relation for the homogeneous IGM contribution to the DM related to the FRB redshift ( $z$ ) as (Zheng et al. 2014; Deng \& Zhang 2014),

$\operatorname{DM}_{\mathrm{IGM}}(z)=\frac{3 c H_{0} \Omega_{\mathrm{b}} f_{\mathrm{IGM}}}{8 \pi G m_{p}} \times \int_{0}^{z} \frac{f_{e}(z)(1+z) d z}{\sqrt{\Omega_{m}(1+z)^{3}+\Omega_{\Lambda}}}$

under the assumption of a flat Universe.

We assume that hydrogen is fully ionized at $z=6$ (i.e. $\chi_{e, \mathrm{H}}=1$ and $\left.\chi_{e, \text { HeII }}=1\right)$ and that the epoch of HeII reionization occurs at $z=3$ in sharp transition (i.e. $\chi_{e, \mathrm{He}}=1, \chi_{e, \mathrm{HeII}}=0$ and $\chi_{e, \mathrm{HeIII}}=1$ ) (Sokasian et al. 2002; Gao et al. 2014; La Plante et al. 2017). It is important to note that we assume the transition to have occurred at the same time across the entire Universe.

\subsection{DM contribution from ISM in the host galaxy}

Knowledge of the DM contribution from the host galaxy is essential to disentangle the amount contributed by the IGM from the total 

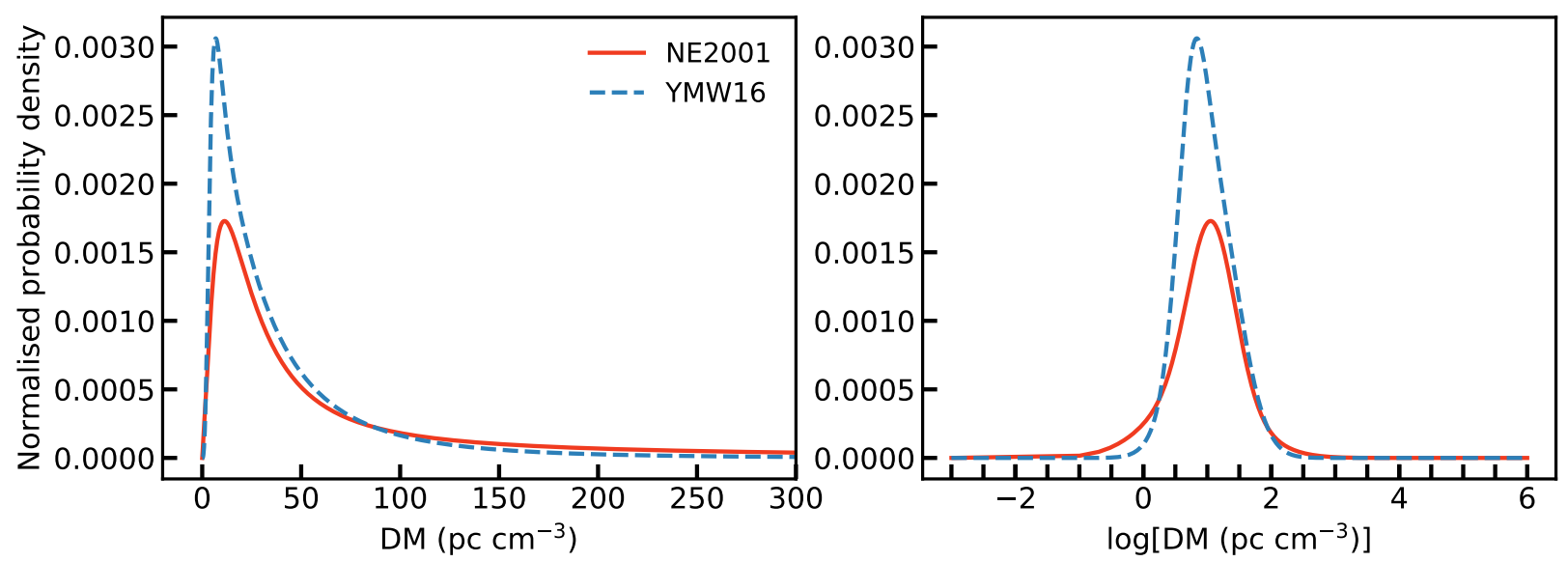

Figure 1. Normalised probability density distributions of the 'all galaxies' sample from (Luo et al. 2018) for the NE2001 and YMW16 electron density maps in linear (left panel) and log scales (right panel). The two curves have been normalised to equal areas.

observed DM. Luo et al. (2018) model the probability distributions of DM contributions for early-type galaxies (ETGs), late-type galaxies (LTGs) and a combination of both types of galaxies called all galaxies (ALGs). The MW and M87 galaxies are chosen to be templates for LTGs and ETGs respectively since their electron density profiles and $\mathrm{H} \alpha$ luminosities are well studied (Cavagnolo et al. 2009; Cordes \& Lazio 2003; Yao et al. 2017). For each of the template galaxies, $10^{6}$ FRBs are simulated at randomly chosen positions following a Galactic stellar distribution (e.g. Young 1976). A detailed modelling of the stellar distribution and the gas fractions can be found in Luo et al. (2018). The FRBs in their model are generated over the full-sky with various line-of-sight inclination angles of their host galaxies using a Monte Carlo method, and the DM probability density function (PDF) is calculated by integrating the electron density along the chosen line-of-sight out to the edge of the galaxy. An important assumption made by Luo et al. (2018) is that all galaxies, dwarfs and giants alike, are equally likely to host FRBs due to the uncertainty in their origins. A consequence of this assumption is that low mass galaxies are more likely to be FRB hosts (as is the case for the repeater FRB 121102), due to simply to their much higher numbers. The zero-redshift rest-frame DM distribution function of the simulated ETGs and LTGs (their Figure 3) are calculated by scaling a $\mathrm{H} \alpha$ luminosity from a luminosity function and an effective galaxy radius from an $r$-band luminosity function to the $\mathrm{H} \alpha$ luminosity and $r$-band luminosity of the template. The resulting PDF of the sample of ALGs is similar to that of the LTGs due to their dominance $(76.3 \%)$ in the sample compared to the ETGs (23.7\%) (Luo et al. 2018).

Luo et al. (2018) present two samples of LTGs modelled after the NE2001 (Cordes \& Lazio 2003) and YMW16 (Yao et al. 2017) Galactic electron density maps from their Monte Carlo method. As a result we adopt the ensemble zero-redshift rest-frame DM PDFs of both ALG samples (NE2001 and YMW16) shown in Figure 1 in our simulations. Galaxies whose electron density distribution follows that of the NE2001 model are seen to produce $\sim 14$ times more FRBs with DMs greater than the $3 \sigma$ value of the YMW16 model (see Figure 1). This is supported by the fact that unlike the NE2001 model the YMW16 model includes no over-dense regions, thereby yielding a much lower average electron density (Yao et al. 2017).

\subsection{DM contribution from progenitor environment}

Despite the association of FRB 121102 with a dwarf galaxy (Chatterjee et al. 2017; Tendulkar et al. 2017; Marcote et al. 2017) the nature of the source producing the FRB is still under debate. Compact objects, particularly neutron star models at cosmological distances, are favoured as FRB progenitors due to their ability to account for the millisecond timescales and observed polarization features. In this case, sources embedded in pulsar wind nebulae or supernova remnants, or near HiI regions will be associated with high electron densities. But the $\mathrm{DM}_{\text {source }}$ contribution is restricted by the condition that the plasma frequency should not exceed the radiation frequency thereby allowing the free propagation of radiation. This limit on the density of the region local to the source along with the cosmological frequency-shift for the range of redshifts of interest in these simulations make the contributions likely to be small. Due to the large uncertainty in the progenitor we do not factor in a value for $\mathrm{DM}_{\text {source }}$.

\section{ANALYSES AND RESULTS}

In our simulations we model two different Universes, one in which HeII reionization occurs in sharp transition at $z=3$ and the other in which it did at $z=6$, represented respectively by the solid and dashed lines in Figure 2. Assuming that FRBs are detected out to $z \sim 6$, we draw $N_{\text {FRBs }}$ from a Gaussian redshift distribution centred on $z=3$ with a $1 \sigma$ spread of $z=1$ fassuming a flat $\Lambda \mathrm{CDM}$ cosmology with the parameters given earlier in Section 2.2. The Gaussian is intended to represent the typical distribution of DMs one gets in practice in a survey at a given telescope. A Gaussian is not a very good approximation to the shape of the DM distribution it typically has a longer tail to high DM than a Gaussian (Xu \& Han 2015; Caleb et al. 2016; Walker et al. 2018) - but a full blown model in which FRBs are simulated with all the selection effects included out to $z=10$, with unknown certain assumptions needing to be made about the numbers (or even the existence) of FRBs at higher $z$ is beyond the scope of this paper. The overall Gaussian shape of the DM distribution is affected by (1) the sensitivity of the survey - higher sensitivity yields higher DM events (2) the frequency and time resolution of the survey - higher frequency resolution allows FRBs to be probed to higher DMs (3) the area of the survey - larger area surveys catch more low DM FRBs (presently, as they typically 

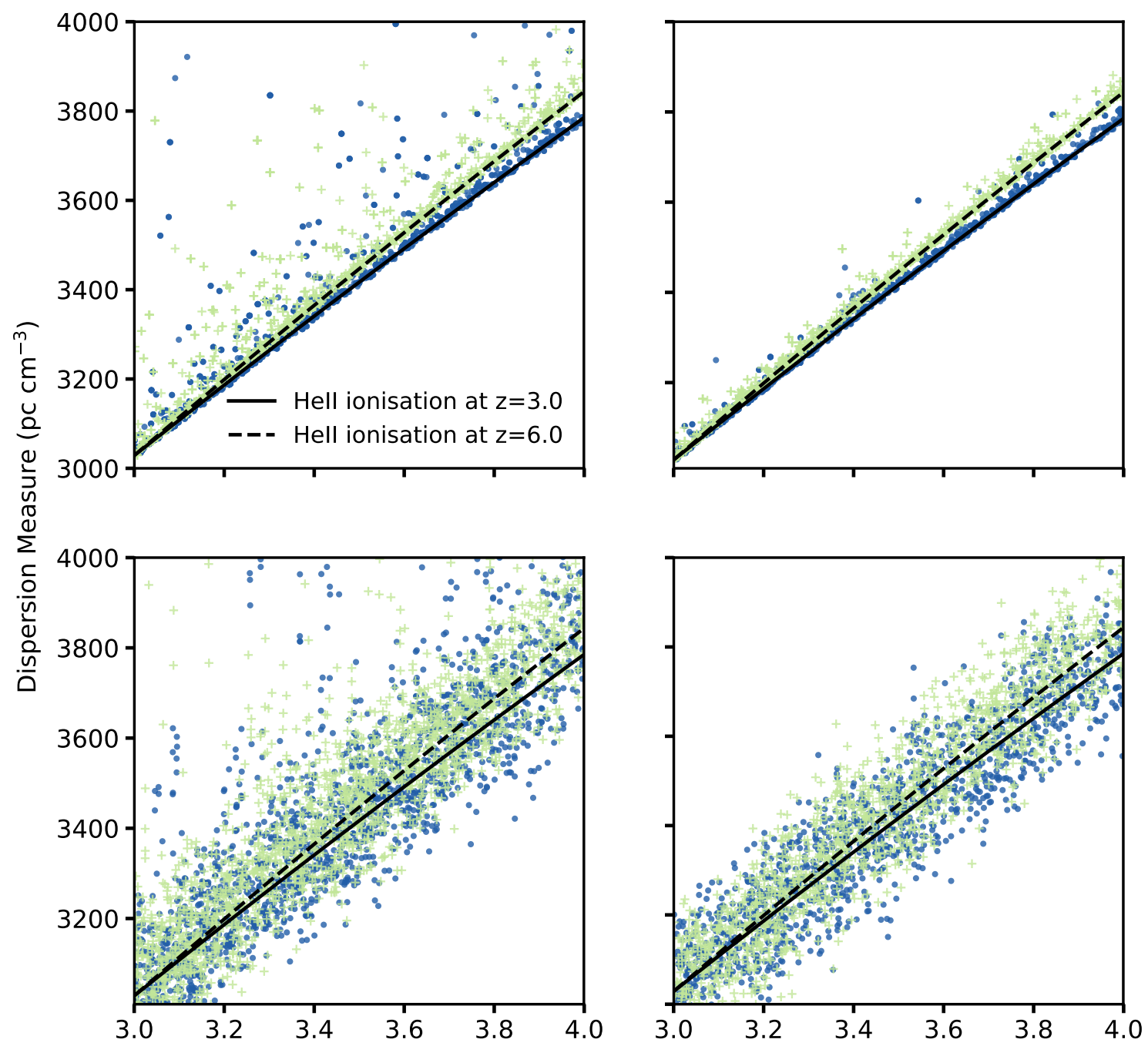

Redshift

Figure 2. Distribution of FRB DMs as a function of redshift. The dashed and solid lines represent two different model Universes in which the HeII reionization occurred at $z=3$ and one in which it occurred at $z=6$. The squares and dots represent the MW and host corrected observed DM values for each of the models (solid and dashed) from Equation 3. The top row panels (top left: Ne2001, top right: YMW16) represent FRBs propagating through a homogeneous IGM and the bottom row panels (bottom left: NE2001, bottom right: YMW16) represent propagation through an inhomogeneous IGM. The DM due to the local environment surrounding the progenitor has not been modelled.

have lower sensitivity as well, but this is changing rapidly with the CHIME (Bandura et al. 2014) and MeerKAT (Stappers 2016) coming on-line). As we aim to keep our model simple, we use a Gaussian distribution. Each FRB at its chosen redshift is assigned a corresponding $\operatorname{DM}_{\mathrm{IGM}}(z)$ from Equation 7. We draw a random host galaxy DM from the zero-redshift rest-frame DM PDF and scale it to the redshift of the simulated FRB based on the star formation rate at that redshift (Hopkins \& Beacom 2006) given by,

$\dot{\rho}_{*}(z)=\frac{0.017+0.13 z}{1+(z / 3.3)^{5.3}} M_{\odot} \mathrm{yr}^{-1} \mathrm{Mpc}^{-3}$.

Figure 2 shows the results of our simulations for $3 \leq z \leq 4$. As detailed in Section 2.3, FRBs following the NE2001 model electron density distribution exhibit a larger scatter in their DMs. For each of the host DM models (NE2001, YMW16) we perform a 2sample Kolmogorov-Smirnoff (KS) test to determine whether we can distinguish between FRBs with $\mathrm{DM}_{\mathrm{IGM}}$ contributions when HeII reionization happened at $z=3$ and $z=6$. A $p$-value of $<0.05$ is our criterion for deciding if the two distributions of FRBs differ. If the Galactic contribution is drawn from the YMW16 model, we require $\sim 950 \mathrm{FRB}$ events in the range $3 \leq z \leq 4$ (see Figure 2) with measured redshifts to distinguish between a Universe in which HeII reionization that occurred at $z=3$ and one in which it occurred at $z=6$ at the $95 \%$ confidence level. For the NE2001 model we require $\sim 1300 \mathrm{FRB}$ events to distinguish between our two simulated models at the same confidence level due to the comparatively larger scatter in host DMs. We emphasize that all these galaxies are required to have measured redshifts and reasonably good corrections $(\sim 50 \%)$ for the host galaxy DM.

We have so far only considered the effect and contribution from a diffuse homogeneous IGM on the observed DM. This however is likely to be too simple a model, due to the presence of free electrons in the halos of potential intervening galaxies. McQuinn 
(2014) simulate the sightline-to-sightline variation in the observed DMs for different galaxy halo mass models (their Figure 1) and estimate the standard deviation in mean value of $\mathrm{DM}_{\mathrm{IGM}}$ to be $180-400 \mathrm{pc} \mathrm{cm}^{-3}$ in the range $0.1 \leq z \leq 1.5$. The halo model in which the distribution of baryons traces dark matter results in the largest dispersion of $400 \mathrm{pc} \mathrm{cm}^{-3}$ from the mean expected value, at $z=1$. Since the curve appears to plateau at $z>1.2$, we model the fluctuations in $\mathrm{DM}_{\mathrm{IGM}}$ due to the halos of intervening galaxies as a Gaussian centred on the mean value of $\mathrm{DM}_{\mathrm{IGM}}$ at that redshift with a $3 \sigma$ spread of $400 \mathrm{pc} \mathrm{cm}^{-3}$. The results are shown in Figure 2. We once again perform a 2-sample KS-test between the two distributions. We require at least 1350 (YMW16) and $\sim 1860$ (NE2001) FRB events with redshift associations to distinguish between the two simulated models of the Universe at the $95 \%$ confidence level. We also consider how well we could constrain the epoch of HeII reionization. We estimate that for a given sample of FRBs between $3 \leq z \leq 5, \sim 5700$ FRBs with measured redshifts are required to distinguish between a HeIr reionization that took place at $z=3$ and $z=3.5$.

\section{DISCUSSION AND CONCLUSION}

In this paper we model the observed DMs of FRBs as a function of redshift to determine if we can measure the epoch of HeII reionization purely based on the DMs. Our first simulations are based on a simple model for the IGM which assumes a homogeneous electron density distribution at all redshifts. The host galaxies of the FRBs have DMs modelled using the results of Luo et al. (2018). We ignore the DM contribution local to the FRB's environment (i.e. surrounding the source) due to the as yet quite uncertain nature of the progenitors and due to the likely dilution of its effect by the $(1+z)$ cosmological frequency-shift. In such an uncomplicated scenario we find we require of order $1100 \mathrm{FRBs}$ with measured redshifts and DMs in the range $3 \leq z \leq 4$ to distinguish a Universe in which HeII reionization occured at $z=3$ from one in which it occurred at $z=6$.

According to McQuinn (2014) the values of DM IGM for two different FRBs at similar redshifts could vary considerably depending on the inhomogeneities along the line-of-sight such as the halos of intervening galaxies, thereby making the case more complicated. The standard deviation around the mean $\operatorname{DM}_{\mathrm{IGM}}(\mathrm{z})$ is $180-400$ pc $\mathrm{cm}^{-3}$ for $0.5<z<1$ depending on the baryon distribution in the different Galactic halo models following different gas profiles (McQuinn 2014). We adopt the sightline dependent variation in DM for the galaxy halo model in which the baryons trace the dark matter halo profile above a threshold of $10^{10} M_{\odot}$. We model the evolution of the DM contribution from the halos of intervening galaxies with redshift, as a normal distribution with a $3 \sigma$ scatter of $400 \mathrm{pc} \mathrm{cm}^{-3}$. Even in such an extreme case with uncertainties in both DM $_{\text {IGM }}$ and $\mathrm{DM}_{\text {host }}$ we are still able to distinguish between our two models of the Universe. However, compared to a homogeneous IGM, in our simulations of an imhomogeneous IGM we need at least $\sim 1600$ FRBs with associated redshifts to determine when the epoch of reionization occurred. We also estimate of order $\sim 5700$ FRBs are needed in the range $3 \leq z \leq 5$ to distinguish between a HeII that took place at $z=3$ and $z=3.5$ at the $95 \%$ confidence level.

The redshifts associated with FRBs require localisations better than a few arcsec of the FRB themselves, or possibly via radio afterglows, if they occur. High spatial resolution is required to localise sufficiently well to perform optical and other multiwavelength follow-ups in order to measure the redshift of the host galaxy. With radio interferometers like the Australian Square Kilometre Array Pathfinder (ASKAP; Bannister et al. 2017) and UTMOST (Bailes et al. 2017) currently doing such FRB searches, and MeerKAT (Stappers 2016) coming online, it is not unreasonable to expect thousands of FRBs to have sufficiently accurate localisations within a few years.

Major caveats pertain to following up such large numbers of FRBs. To probe redshifts beyond $z \approx 3$, FRBs with DMs $\gtrsim 3000$ need to be found, which requires sensitive instruments with high frequency resolution in order to avoid the effects of dispersion smearing (only one FRB is currently known with a DM of more than 2000 $\mathrm{pc} \mathrm{cm}^{-3}$ ). Zhang (2018) has shown that high sensitivity telescopes with large apertures (e.g. FAST) are capable of finding FRBs out to redshifts as high as $z \sim 15$, assuming their progenitors actually form at such early times. Follow-up of thousands of localised host galaxies for $z \gtrsim 3$ requires high localisation accuracy, due to the source density on the sky (Conselice et al. 2016; Eftekhari \& Berger 2017) and is strongly dependent on the typical host galaxies for FRBs, which remains to be determined. While spectroscopic redshifts would be ideal, we note that photometrically obtained redshifts could be of sufficient quality : photometric redshifts show a scatter $d z /(1+z)$ (Straatman et al. 2016) of only $\approx 0.03$ (Ilbert et al. 2006) around the spectroscopic-photometric redshift relation, which corresponds to a an additional scatter of order $30 \mathrm{pc} \mathrm{cm}^{-3}$ in the redshift-DM relation, smaller than the scatter in our modeling (due to the host galaxy properties and/or inhomegeneities in the IGM). Despite these caveats, the combination of next generation radio telescopes like the Square Kilometer Array ${ }^{1}$ and optical telescopes like the Large Synoptic Survey Telescope ${ }^{2}$ and the James Webb Space Telescope ${ }^{3}$ could transform our understanding of the host galaxy DM contributions and our ability to study the epoch of HeII reionization.

\section{ACKNOWLEDGEMENTS}

MC would like to thank Rui Luo, Kejia Lee, Charles Walker and Themiya Nanayakkara for useful discussions. MC and BWS acknowledge funding from the European Research Council (ERC) under the European Union's Horizon 2020 research and innovation programme (grant agreement No 694745). CF acknowledges financial support by the Beckwith Trust.

\section{REFERENCES}

Bailes M., et al., 2017, Publ. Astron. Soc. Australia, 34, e045

Bandura K., et al., 2014, in Ground-based and Airborne Telescopes V. p. 914522 (arXiv: 1406.2288), doi:10.1117/12.2054950

Bannister K. W., et al., 2017, ApJ, 841, L12

Caleb M., Flynn C., Bailes M., Barr E. D., Hunstead R. W., Keane E. F., Ravi V., van Straten W., 2016, MNRAS, 458, 708

Cavagnolo K. W., Donahue M., Voit G. M., Sun M., 2009, ApJS, 182, 12

Chatterjee S., et al., 2017, Nature, 541, 58

Conselice C. J., Wilkinson A., Duncan K., Mortlock A., 2016, ApJ, 830, 83

Cordes J. M., Lazio T. J. W., 2003, preprint, (arXiv:0207156)

DeBoer D. R., et al., 2017, PASP, 129, 045001

Deng W., Zhang B., 2014, ApJ, 783, L35

\footnotetext{
1 https://www.skatelescope.org/

2 https://www.lsst.org/

3 https://www.jwst.nasa.gov/
} 
Dolag K., Gaensler B. M., Beck A. M., Beck M. C., 2015, MNRAS, 451, 4277

Eftekhari T., Berger E., 2017, ApJ, 849, 162

Fan X., Narayanan V. K., Strauss M. A., White R. L., Becker R. H., Pentericci L., Rix H.-W., 2002, AJ, 123, 1247

Fukugita M., Hogan C. J., Peebles P. J. E., 1998, ApJ, 503, 518

Gao H., Li Z., Zhang B., 2014, ApJ, 788, 189

Hopkins A. M., Beacom J. F., 2006, ApJ, 651, 142

Ilbert O., et al., 2006, A\&A, 457, 841

Ioka K., 2003, ApJ, 598, L79

Keane E. F., 2018, Nature Astronomy, 2, 865

La Plante P., Trac H., Croft R., Cen R., 2017, preprint, (arXiv:1710.03286)

Luo R., Lee K., Lorimer D. R., Zhang B., 2018, MNRAS, 481, 2320

Macquart J. P., et al., 2015, Advancing Astrophysics with the Square Kilometre Array (AASKA14), p. 55

Marcote B., et al., 2017, ApJ, 834, L8

McQuinn M., 2014, ApJ, 780, L33

McQuinn M., Lidz A., Zaldarriaga M., Hernquist L., Hopkins P. F., Dutta S., Faucher-Giguère C.-A., 2009, ApJ, 694, 842

Parsons A. R., et al., 2014, ApJ, 788, 106

Planck Collaboration et al., 2016, A\&A, 594, A13

Shull J. M., Smith B. D., Danforth C. W., 2012, ApJ, 759, 23

Singh S., et al., 2018, ApJ, 858, 54

Sokasian A., Abel T., Hernquist L. E., 2002, ArXiv Astrophysics e-prints,

Stappers B., 2016, in Proceedings of MeerKAT Science: On the Pathway to the SKA. 25-27 May, 2016 Stellenbosch, South Africa (MeerKAT2016). p. 10

Straatman C. M. S., et al., 2016, ApJ, 830, 51

Syphers D., et al., 2009, The Astrophysical Journal, 690, 1181

Syphers D., Anderson S. F., Zheng W., Meiksin A., Schneider D. P., York D. G., 2012, The Astronomical Journal, 143, 100

Tendulkar S. P., et al., 2017, ApJ, 834, L7

Tingay S. J., et al., 2013, Publ. Astron. Soc. Australia, 30, e007

Walker C. R. H., Ma Y.-Z., Breton R. P., 2018, arXiv e-prints,

Xu J., Han J. L., 2015, preprint, (arXiv: 1504.00200)

Yao J. M., Manchester R. N., Wang N., 2017, ApJ, 835, 29

Young P. J., 1976, AJ, 81, 807

Zaroubi S., 2013, in Wiklind T., Mobasher B., Bromm V., eds, Astrophysics and Space Science Library Vol. 396, The First Galaxies. p. 45 (arXiv : 1206.0267), doi:10.1007/978-3-642-32362-1_2

Zhang B., 2018, ApJ, 867, L21

Zheng Z., Ofek E. O., Kulkarni S. R., Neill J. D., Juric M., 2014, ApJ, 797, 71

Zhou B., Li X., Wang T., Fan Y.-Z., Wei D.-M., 2014, Phys. Rev. D, 89, 107303

van Haarlem M. P., et al., 2013, A\&A, 556, A2

This paper has been typeset from a $\mathrm{T}_{\mathrm{EX}} \mathrm{X} \mathrm{L} \mathrm{T} \mathrm{EX}$ file prepared by the author. 